\title{
Dysfunctional Coagulation in COVID-19: From Cell to Bedside
}

Jie Wang · Ardan M. Saguner · Jiaqi An · Yuye Ning · Yang Yan ·

Guoliang Li (1)

Received: April 29, 2020 / Published online: June 5, 2020

(c) Springer Healthcare Ltd., part of Springer Nature 2020

\section{ABSTRACT}

Severe acute respiratory syndrome coronavirus 2 (SARS-CoV-2) causes coronavirus disease 2019 (COVID-19), which can induce multisystem disease. Human angiotensin-converting enzyme 2 (ACE2) widely expressing in arterial and venous endothelial cells and arterial smooth muscle cells has been identified as a functional receptor for SARS-CoV-2. Dysfunction of ACE2 leads to abnormal activation of the renin-angiotensin system and a systemic

Digital Features To view digital features for this article go to https://doi.org/10.6084/m9.figshare.12369890.

J. Wang · Y. Ning · G. Li ( $\varangle)$

Department of Cardiovascular Medicine, The First Affiliated Hospital of Xi'an Jiaotong University, 277 Yanta West Road, Xi'an 710061, China

e-mail: liguoliang_med@163.com

J. Wang

Department of Hematology, The First Affiliated Hospital of Xi'an Jiaotong University, 277 Yanta West Road, Xi'an 710061, China

\section{A. M. Saguner}

Department of Cardiology, University Heart Center Zurich, Rämistrasse 100, 8091 Zurich, Switzerland

\section{J. An · Y. Ning}

Stroke Centre and Department of Neurology, The First Affiliated Hospital of Xi'an Jiaotong University, 277 Yanta West Road, Xi'an 710061, China endotheliitis that may relate to abnormal coagulation and sepsis. Meanwhile, innate immune response and inflammation activation participate in dysfunctional coagulation. Previous research indicated that dysfunctional coagulation was one of the important risk factors accountable for a high risk of severe disease and death in patients with COVID-19. Understanding the possible mechanisms of dysfunctional coagulation and appropriate anticoagulation therapeutic strategies are important to prevent disease deterioration and reduce fatality rates during the ongoing COVID-19 pandemic.
J. An

Department of Neurology, Massachusetts General Hospital, 55 Fruit St, Lunder 644, Boston, MA 02114, USA

Y. Yan $(\bowtie)$

Department of Cardiovascular Surgery, The First Affiliated Hospital of Xi'an Jiaotong University, 277 Yanta West Road, Xi'an 710061, Shaanxi, China e-mail: yangyan3@xjtu.edu.cn 
Keywords: ACE2; COVID-19; Dysfunctional coagulation; SARS-CoV-2

\section{Key Summary Points}

Why carry out this study?

Dysfunctional coagulation is one of the important risk factors accountable for the high risk of severe disease and death in patients with coronavirus disease 2019 (COVID-19) infection. Severe acute respiratory syndrome coronavirus 2 (SARS-CoV-2) involves potentially deleterious processes in hemostasis/coagulation and the inflammation system.

This article aims to discuss the possible mechanisms underlying dysfunctional coagulation during SARS-CoV-2 infection.

\section{What was learned from the study?}

Abnormal activation of the reninangiotensin system (RAS) and a systemic endotheliitis caused by dysfunction of angiotensin-converting enzyme 2 (ACE2), the innate immune response and inflammation activation participate in dysfunctional coagulation.

To propose an appropriate anticoagulation therapeutic regimen and reduce the fatality rate during the ongoing COVID19 pandemic.

\section{INTRODUCTION}

As of 19 May 2020, there were $>4,800,000$ confirmed cases of coronavirus disease 2019 (COVID19) worldwide with $>300,000$ deaths. Severe acute respiratory syndrome-coronavirus 2 (SARSCoV-2) is identified as the pathogen of COVID-19 [1]. SARS-CoV-2 shares about $80 \%$ homology with that of severe acute respiratory syndrome coronavirus (SARS-CoV) [2]. SARS-CoV-2 enters and infects cells by using the receptor of human angiotensin-converting enzyme 2 (ACE2) (Fig. 1), and its spike protein binds to their common host cell receptor at least ten times more tightly than the corresponding spike protein of SARS-CoV $[3,4]$. ACE2 is expressed widely in various tissues. This may cause multiorgan dysfunction, including the vascular system, heart, kidneys, liver, retina, enterocytes of the intestines and circumventricular organs of the central nervous system, upper airway tract, alveolar (type II) epithelial cells of the lungs and pulmonary vasculature (Fig. 2) [5-8].

The dysfunction of endothelial cells interacts with the inflammation due to coronavirus infection and may lead to abnormal coagulation and sepsis, indicating a poor prognosis in patients with COVID-19 [9]. The pooled frequency of thrombocytopenia is $11.1 \%$ [10], and higher levels of D-dimers seem to be more common in patients with COVID-19, from $46.4 \%$ to $97.1 \%$ [11-13], especially in the severe cases [14-17]. Increased coagulation eventually causes multiorgan thromboembolism and death, which is further confirmed by pathologic evidence from fatal cases subject to necropsy [18]. Adults $\geq$ 60 years, especially those with preexisting heart disease, lung disease, hypertension, diabetes and cancer, are very vulnerable to SARS-CoV-2 infection and often develop severe disease. Dysfunctional coagulation is considered to constitute one of the important risk factors accountable for this high risk of severe disease and death $[19,20]$. Therefore, the immediate priority is to unveil the possible causes of dysfunctional coagulation and recommend an accurate anticoagulation therapeutic regimen to protect patients from aggravation and death $[21,22]$. This article is based on previously conducted studies and does not contain any studies with human participants or animals performed by any of the authors.

\section{COVID-19 INFECTION AND COAGULATION FUNCTION}

Coagulation is an extremely well-organized process that involves the interaction of three key components: endothelial cells, platelets and coagulation factors. In severe infection, 


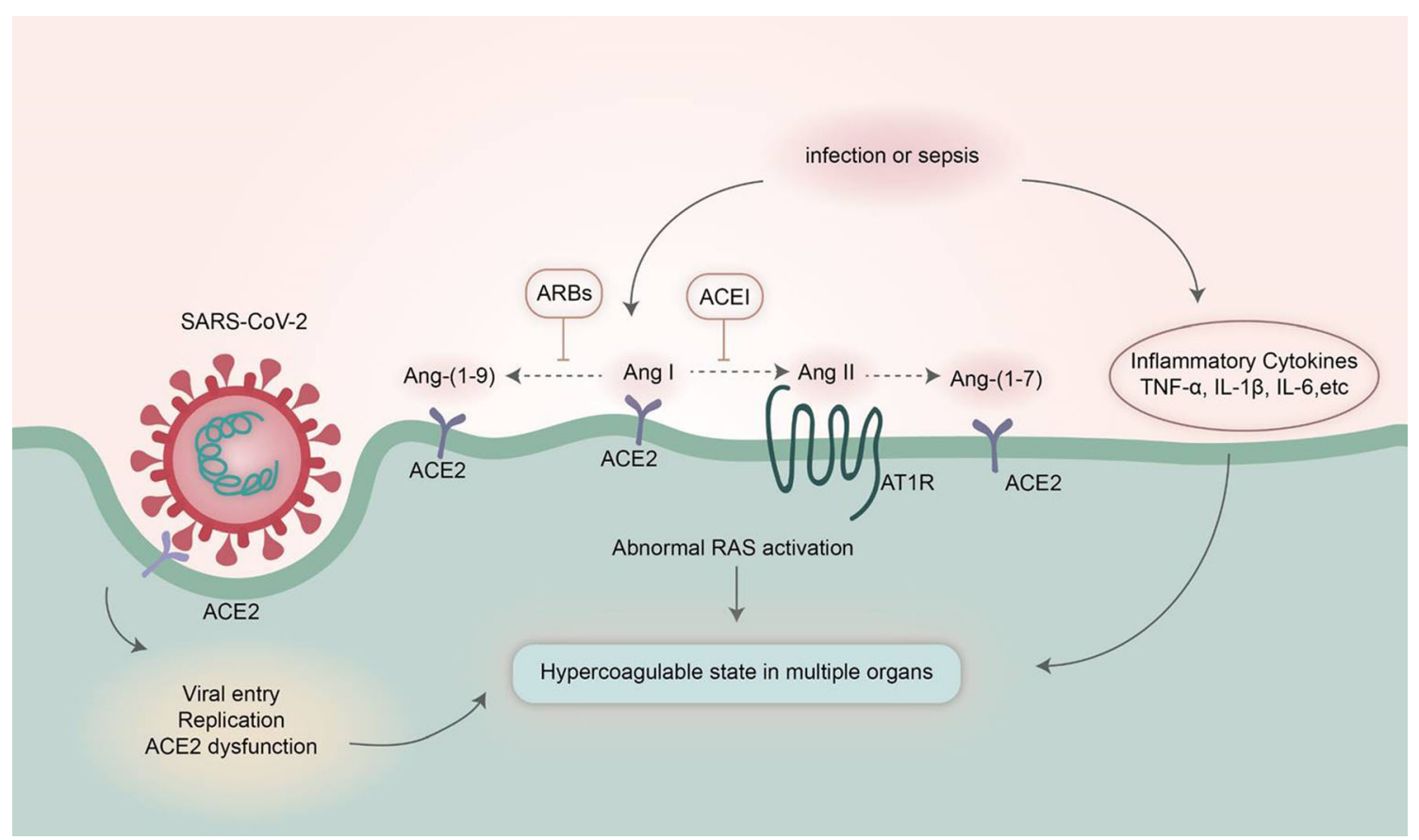

Fig. 1 Mechanisms of dysfunctional coagulation in COVID-19. Dysfunction of ACE2 leads to abnormal RAS activation, which promotes platelet adhesion and aggregation following the invasion of SARS-CoV-2. Meanwhile, inflammatory cytokines activate the coagulation cascade. COVID-19 coronavirus disease 2019, SARS$\mathrm{CoV}-2$ severe acute respiratory syndrome-coronavirus 2,

coagulation is activated, and there is an increased risk of the development of excessive consumption of coagulation factors with attendant disseminated intravascular coagulation, which increases the fatality rates [23]. Considerable evidence shows that patients contracting COVID-19 manifest abnormal coagulation in both clinical presentation and laboratory examination $[19,20]$. Though the specific mechanisms are still unclear, SARSCoV-2 obviously involves potentially deleterious processes in hemostasis/coagulation and inflammation.

\section{DYSFUNCTIONAL ACE2}

ACE2 is an important part of the renin-angiotensin system (RAS), which has an opposite effect to ACE. The RAS is composed of two axes including ACE-angiotensin II (Ang II)-
ACE2 angiotensin-converting enzyme 2, Ang $I$ angiotensin I, Ang II angiotensin II, Ang-(1-9) angiotensin-(1-9), Ang(1-7) angiotensin-(1-7), ATIR angiotensin II type 1 receptor, $R A S$ renin-angiotensin system, $T N F-\alpha$ tumor necrosis factor- $\alpha, I L-1 \beta$ interleukin-1 $\beta, I L-6$ interleukin-6, $A C E I$ angiotensin-converting enzyme inhibitors, $A R B$ angiotensin II receptor blocker

angiotensin II type 1 receptor (AT1R) and ACE2Ang (1-7)-Mas receptor (MasR). The first axis of RAS elevates reactive oxygen species and superoxide levels, impairing endothelial function and microcirculation. The ACE2-Ang (1-7)MasR axis counteracts the function of the ACEAng II-AT1R axis, which decreases inflammation and produces vasodilatation [24]. Dysfunction of ACE2 leads to abnormal activation of the ACE-Ang II-AT1R axis, which subsequently promotes platelet adhesion and aggregation and enhances the risk of thromboembolism in multiple organs including the lungs, brain, heart, kidney, etc.

\section{INNATE IMMUNE RESPONSE}

The coagulation system acts as a host defense response to protect the human body from viral invasion or injury. The activation of hemostatic 

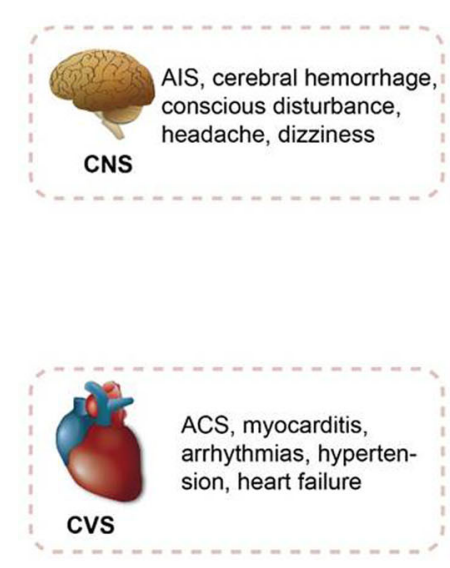

Fig. 2 Multiorgan injuries in COVID-19. COVID-19 coronavirus disease 2019, CNS central nervous system, $A I S$ acute ischemic stroke, $C V S$ cardiovascular system, $A C S$

processes induced by virus invasion may work as an immune system to eliminate the etiologic agent inside the clot [25]. In fact, the regulation of coagulation and innate immunity is correlated and intertwined because they share many common pathways in response to viral invasion and injury, such as the function of tissue factor in the initiation of procoagulation, proinflammation and the host immune response [26].

\section{INFLAMMATORY FACTOR STORM}

Previous data indicate that patients with COVID-19 pneumonia show a cytokine storm at the very early stage of the disease course, and the most recent pathologic evidence from autopsies of patients dying from COVID-19 also supports this idea [18].
涪:

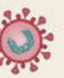

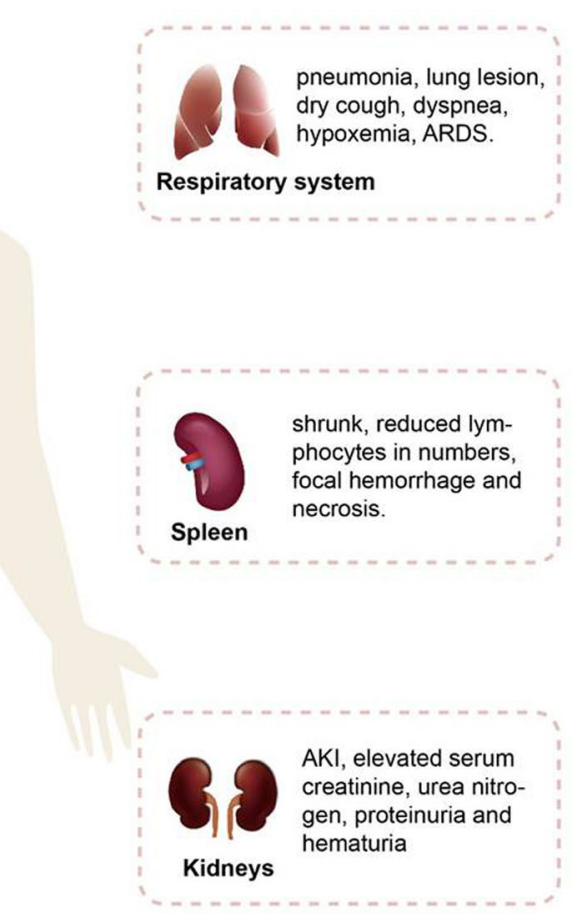
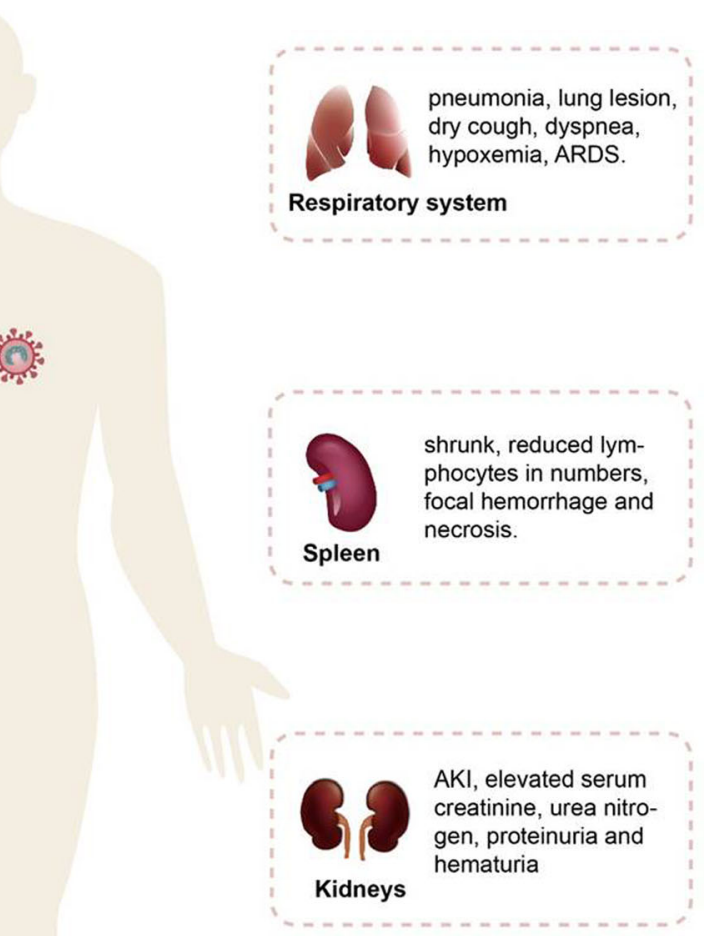

acute coronary syndrome, $A L T$ alanine transaminase, $A S T$ aspartate transaminase, $A R D S$ acute respiratory distress syndrome, $A K I$ acute kidney injury

Elevated levels of inflammation-related cytokines, including interleukin-2 (IL-2), interleukin-6 (IL-6), interleukin-7, interleukin-8, interleukin-10 and tumor necrosis factor (TNF), were found in patients with COVID-19 $[18,27,28]$ and correlated with disease severity [29]. Endothelial cell infection and endotheliitis play a critical role in the pathogenesis of SARSCoV-2. Inflammation due to virus infection aggravates various proinflammatory cytokines such as TNF- $\alpha$, IL-1 $\beta$ and IL-6, which increase the expression of tissue factor and von Willebrand factor from endothelial cells and monocytes, promoting platelet aggregation and initiating the clotting cascade. Besides, proinflammatory cytokines can also suppress the synthesis of anticoagulants and fibrinolysis by downregulating thrombomodulin and endothelial protein $C$ receptor and upregulating plasminogen activator inhibitor-1 [30]. 
Altogether, current evidence has shown the potential of the intimate interconnection with inflammatory disorders, hypercoagulation and excessive immunity following SARS-CoV-2 invasion in dysfunctional coagulation. The stimulation of an immune response and proinflammation activate the coagulation cascade, and blood clotting in turn orchestrates the pathway of an excessive inflammatory response.

\section{PREVENTION AND TREATMENT}

The lungs are the organs most affected by COVID-19, and pneumonia-like symptoms are the most common clinical manifestation in infected patients with moderate-to-severe cases, some of whom may experience a sudden worsening of clinical conditions with rapid deterioration into respiratory failure and multiple organ dysfunction syndromes, especially in patients $\geq 60$ years old with underlying chronic diseases. The therapeutic regimen of COVID-19 varies based on the severity, including general and supportive treatment. As this is a viral pneumonia, antiviral therapies are experimental, and targeted therapeutic regimens against SARS-CoV-2 are still under investigation. However, antibacterial drugs should be used prudently.

In fighting SARS-CoV-2, the immediate priority is to balance the immune response, inflammation activation and hypercoagulation, as disorders of these three systems usually indicate poor outcomes. First, healthcare workers should carefully screen their patients, especially those with underlying diseases who are at high risk of endotheliitis induced by SARS-CoV-2 infection; a systemic endotheliitis may be the first step of viral invasion [9]. Second, dynamic monitoring of coagulation and inflammationrelated biomarkers are important to predict outcomes and adjust the anticoagulant and antiplatelet strategies in a timely manner. Third, in severe cases, some methods to remove the cytokines and clotting factors should be considered, such as continuous renal replacement therapies, immunotherapy and artificial liver support systems. Finally, avoiding drug-drug interactions contributory to hypercoagulation should be emphasized, especially since a variety of substances are now being used in combination in clinical trials.

\section{PROSPECTS AND CHALLENGES}

COVID-19 infection disrupts the balance of coagulative and fibrinolytic pathways, resulting in an abnormal activation of coagulation and a secondary hyperfibrinolysis, leading to an increased risk of thromboembolism in several organs. Given that the progressive deterioration of coagulation function has an unfavorable effect on the clinical course, it is never too early to start routine monitoring of hemostasis and inflammation tests. Moreover, evidence indicates that anticoagulant treatment was effective when used early in COVID-19 patients [21, 22]. These findings suggest that anticoagulation therapy should be applied as soon as D-dimers increase or prophylactic anticoagulation should be used throughout the whole clinical course unless contraindicated, e.g., in case of acute pneumorrhagia or hematencephalon. The underlined pathologic and physiologic mechanisms of hypercoagulation in patients with COVID-19 should be further explored, which will definitely help design an accurate therapeutic strategy to prevent disease deterioration and reduce fatality rates during the current COVID-19 pandemic.

\section{ACKNOWLEDGEMENTS}

All authors thank Dr. Lv Liu and Miaomiao Liu for sharing their valuable experience in the management of patients with COVID-19 in Wuhan, China, and Dr. Danjun Zhu for helpful comments and discussion of the manuscript.

Funding. This study was supported by the Clinical Research Program of the First Affiliated Hospital of Xi'an Jiaotong University of China (XJTU1AF-CRF-2018-015). The rapid service fee was funded by the authors.

Authorship. All named authors meet the International Committee of Medical Journal 
Editors (ICMJE) criteria for authorship for this article, take responsibility for the integrity of the work as a whole, and have given their approval for this version to be published.

Author Contributions. All authors contributed to drafting the manuscript, reviewing the manuscript content critically, and designing and implementation of this article. Yuye Ning and Yang Yan illustrated and captioned all figures.

Disclosures. Ardan M. Saguner has received lecture honoraria from Boston Scientific and educational grants from Abbott, Bayer, Boston Scientific, Biosense Webster, Biotronik, Medtronic and Pfizer-BMS. He owns shares in Gilead Sciences, Inc. Jie Wang, Jiaqi An, Yuye Ning, Yang Yan and Guoliang Li have nothing to disclose in relation to this article.

Compliance with Ethics Guidelines. This article is based on previously conducted studies and does not contain any studies with human participants or animals performed by any of the authors.

Data Availability. Data sharing is not applicable to this article as no datasets were generated or analyzed during the current study.

\section{REFERENCES}

1. Zhu N, Zhang D, Wang W, et al. A novel coronavirus from patients with pneumonia in China, 2019. N Engl J Med. 2020;382:727-33.

2. Zhou P, Yang XL, Wang XG, et al. A pneumonia outbreak associated with a new coronavirus of probable bat origin. Nature. 2020;579:270-3.

3. Wrapp D, Wang N, Corbett KS, et al. Cryo-EM structure of the 2019-nCoV spike in the prefusion conformation. Science. 2020;367:1260-3.

4. Letko M, Marzi A, Munster V. Functional assessment of cell entry and receptor usage for SARS-CoV2 and other lineage $B$ betacoronaviruses. Nat Microbiol. 2020;5:562-9.

5. Gheblawi M, Wang K, Viveiros A, et al. Angiotensin-converting enzyme 2: SARS-CoV-2 receptor and regulator of the renin-angiotensin system: celebrating the 20th anniversary of the discovery of ACE2. Circ Res. 2020;126:1456-74.

6. Zhou J, Li C, Liu X, et al. Infection of bat and human intestinal organoids by SARS-CoV-2. Nat Med. 2020. https://doi.org/10.1038/s41591-0200912-6.

7. $\mathrm{Hu} \mathrm{Y,} \mathrm{Chen} \mathrm{T,} \mathrm{Liu} \mathrm{M,} \mathrm{et} \mathrm{al.} \mathrm{Positive} \mathrm{detection} \mathrm{of}$ SARS-CoV-2 combined HSV1 and HHV6B virus nucleic acid in tear and conjunctival secretions of a non-conjunctivitis COVID-19 patient with obstruction of common lacrimal duct. Acta Ophthalmol. 2020. https://doi.org/10.1111/aos.14456.

8. Menni C, Valdes AM, Freidin MB, et al. Real-time tracking of self-reported symptoms to predict potential COVID-19. Nat Med. 2020. https://doi. org/10.1038/s41591-020-0916-2.

9. Varga Z, Flammer AJ, Steiger P, et al. Endothelial cell infection and endotheliitis in COVID-19. Lancet. 2020;395:1417-8.

10. Nasiri MJ, Haddadi S, Tahvildari A, et al. COVID-19 clinical characteristics, and sex-specific risk of mortality: Systematic Review and Meta-analysis. medRxiv. 2020:2020.03.24.20042903; doi: https:// doi.org/10.1101/2020.03.24.20042903.

11. Zhang B, Zhou X, Qiu Y, et al. Clinical characteristics of 82 death cases with COVID-19. medRxiv: 2020:2020.02.26.20028191. https://doi.org/10. $1101 / 2020.02 .26 .20028191$.

12. Luo $\mathrm{X}$, Xia H, Yang $\mathrm{W}$, et al. Characteristics of patients with COVID-19 during epidemic ongoing outbreak in Wuhan, China. medRxiv:2020:2020.03. 19.20033175. https://doi.org/10.1101/2020.03.19. 20033175.

13. Guan W-J, Ni Z-Y, Hu Y, et al. Clinical characteristics of coronavirus disease 2019 in China. N Engl J Med. 2020;382:1708-20.

14. Zhang $\mathrm{H}$, Wang $\mathrm{X}, \mathrm{Fu} \mathrm{Z}$, et al. Potential factors for prediction of disease severity of COVID-19 patients. medRxiv:2020:2020.03.20.20039818. https://doi. org/10.1101/2020.03.20.20039818.

15. Shi Q, Zhao K, Yu J, et al. Clinical characteristics of 101 non-surviving hospitalized patients with COVID-19: a single center, retrospective study. medRxiv:2020:2020.03.04.20031039. https://doi. org/10.1101/2020.03.04.20031039.

16. Cao M, Zhang D, Wang Y, et al. Clinical features of patients infected with the 2019 novel coronavirus (COVID-19) in Shanghai, China. medRxiv:2020: 2020.03.04.20030395. https://doi.org/10.1101/ 2020.03.04.20030395. 
17. Zhang G, Hu C, Luo L, et al. Clinical features and outcomes of 221 patients with COVID-19 in Wuhan, China. medRxiv:2020:2020.03.02. 20030452. https://doi.org/10.1101/2020.03.02. 20030452.

18. Wang C, Xie J, Zhao L, et al. Aveolar macrophage activation and cytokine storm in the pathogenesis of severe COVID-19. 2020. https://doi.org/10. 21203/rs.3.rs-19346/v1.

19. Tang N, Li D, Wang X, Sun Z. Abnormal coagulation parameters are associated with poor prognosis in patients with novel coronavirus pneumonia. J Thromb Haemost. 2020;18:844-7.

20. Giannis D, Ziogas IA, Gianni P. Coagulation disorders in coronavirus infected patients: COVID-19, SARS-CoV-1, MERS-CoV and lessons from the past. J Clin Virol. 2020;127:104362.

21. Kollias A, Kyriakoulis KG, Dimakakos E, Poulakou G, Stergiou GS, Syrigos K. Thromboembolic risk and anticoagulant therapy in COVID-19 patients: emerging evidence and call for action. Br J Haematol. 2020. https://doi.org/10.1111/bjh.16727.

22. Tang N, Bai H, Chen X, Gong J, Li D, Sun Z. Anticoagulant treatment is associated with decreased mortality in severe coronavirus disease 2019 patients with coagulopathy. J Thromb Haemost. 2020;18:1094-9.

23. Han H, Yang L, Liu R, et al. Prominent changes in blood coagulation of patients with SARS-CoV-2 infection. Clin Chem Lab Med. 2020. https://doi. org/10.1515/cclm-2020-0188.

24. Yang T, Chen YY, Liu JR, et al. Natural products against renin-angiotensin system for antifibrosis therapy. Eur J Med Chem. 2019;179:623-33.

25. Minasyan H, Flachsbart F. Blood coagulation: a powerful bactericidal mechanism of human innate immunity. Int Rev Immunol. 2019;38:3-17.

26. Delvaeye M, Conway EM. Coagulation and innate immune responses: can we view them separately? Blood. 2009;114:2367-74.

27. Huang C, Wang Y, Li X, et al. Clinical features of patients infected with 2019 novel coronavirus in Wuhan, China. Lancet. 2020;395:497-506.

28. Chen N, Zhou M, Dong X, et al. Epidemiological and clinical characteristics of 99 cases of 2019 novel coronavirus pneumonia in Wuhan, China: a descriptive study. Lancet. 2020;395:507-13.

29. Gong J, Dong H, Xia SQ, et al. Correlation analysis between disease severity and inflammation-related parameters in patients with COVID-19 pneumonia. medRxiv:2020:2020.02.25.20025643. https://doi. org/10.1101/2020.02.25.20025643.

30. Lipinski S, Bremer L, Lammers T, Thieme F, Schreiber S, Rosenstiel P. Coagulation and inflammation. Molecular insights and diagnostic implications. Hamostaseologie. 2011;31(94-102):4. 